The Future of Polar

\title{
Organometallic Chemistry
}

Written in Bio-Based Solvents

\section{and Water}

Joaquin García-Álvarez, ${ }^{*[a]}$ Eva Hevia, ${ }^{*[b]}$ and Vito

Capriati*[c] $^{*}$

Dedication ((optional))

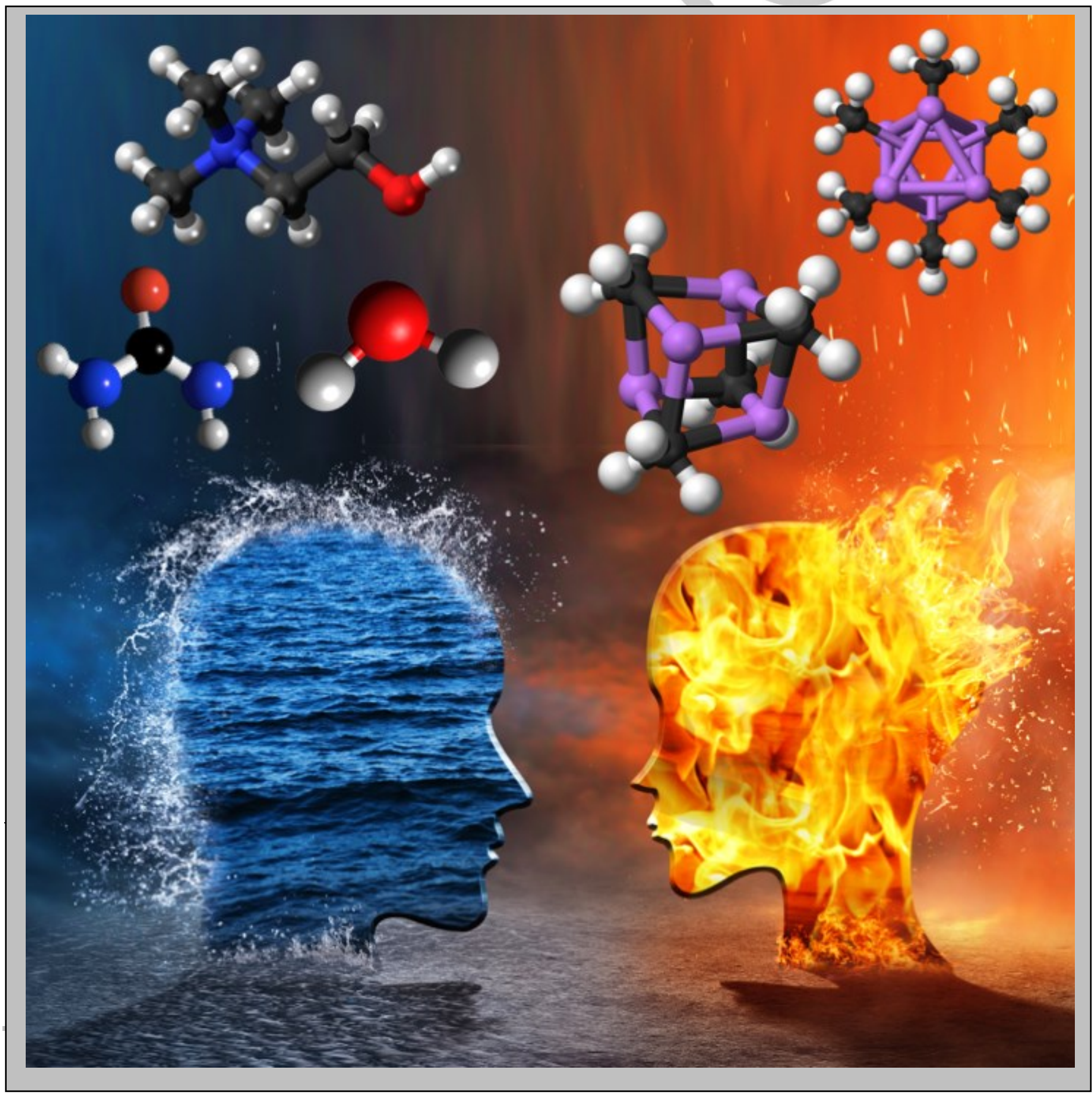


Abstract: There is a strong imperative to reduce the release of volatile organic compounds (VOCs) into the environment, and many efforts are currently being made to replace conventional hazardous VOCs in favour of safe, green and bio-renewable reaction media that are not based on crude petroleum. Recent ground-breaking studies from a few laboratories worldwide have shown that both Grignard and (functionalised) organolithium reagents, traditionally handled under strict exclusion of air and humidity and in anhydrous VOCs, can smoothly promote both nucleophilic additions to unsaturated substrates and nucleophilic substitutions in water and other bio-based solvents (glycerol, deep eutectic solvents), competitively with protonolysis, at room temperature and under air. The chemistry of polar organometallics in the above protic media is a complex phenomenon influenced by several factors, and understanding its foundational character is surely stimulating in the perspective of the development of a sustainable organometallic chemistry.

\section{Introduction}

The Twelve Principles of Green Chemistry published in 1998 have provided useful guidelines and framework for the design of chemical products and processes safer and more sustainable, and for reducing or eliminating either pollution in the chemical industry or the generation and use of hazardous substances. ${ }^{[1]}$ Since then, and similarly to an opening cornucopia, significant research supported by exponential growth of publications has been directed towards the developments of more efficient and environmentally benign and clean technologies with high atom economies. ${ }^{[2]}$ In particular, special efforts have been made to replace traditionally harmful, toxic/carcinogenic petroleum-based volatile organic compounds (VOCs) with "greener", neoteric solvents $\left(\right.$ neoteric $=$ recent, new) ${ }^{[3 a]}$ with properties similar to many existing solvent types. This is because solvents are used in such large quantities in the practice of synthetic organic chemistry in academy and in industry worldwide contributing to over $80 \%$ of the organic waste produced. ${ }^{[3 b, c]}$

Anyway, aside from the standpoint of "greenness", it cannot be disregarded that the chemical and physical properties of solvents also exert considerable influence on the rates of homogeneous/heterogeneous chemical reactions, on the

[a] Dr. Joaquín García-Álvarez

Laboratorio de Compuestos Organometálicos y Catálisis

Departamento de Química Orgánica e Inorganica (IUQOEM)

Instituto Universitario de Química Organometálica "Enrique Moles"

Facultad de Química, Universidad de Oviedo

E-33071, Oviedo, Spain

E-mail: garciajoaquin@uniovi.es

[b] Prof. Eva Hevia

WestCHEM, Department of Pure and Applied Chemistry

University of Strathclyde, Glasgow, G1 1XL (UK)

Email: eva.hevia@strath.ac.uk

[c] Prof. Vito Capriati

Dipartimento di Farmacia-Scienze del Farmaco

Università di Bari "Aldo Moro", Consorzio C.I.N.M.P.I.S.

Via E. Orabona, 4 - I-70125 Bari, Italy

Email: vito.capriati@uniba.it position of chemical equilibria, and overall on the outcome of the reaction itself. ${ }^{[4]}$ In the quest for the right, ideal solvent for any reaction, some important interrogations that future scientific innovations in green chemistry need to answer were recently raised by Paul Anastas (Yale University), the "Father of Green Chemistry", during the XXVI National Congress of the Italian Chemical Society which was held in Paestum (Salerno, Italy) from September 10th to 14th, 2017: will our solvents be designed to be obedient; changing properties as desired upon imparted stimulus? will we use weak forces (such as hydrogen bonding and $\pi$-interactions) as design tools to impart performance properties and control reaction pathways ? $^{[5]}$

In this perspective, the unexpected behaviour and reactivity manifested in recent years by polar organometallic compounds of s-, p-, and d-block elements when used in novel, designed unconventional solvents that offer tunability such as the so-called deep eutectic solvents and ionic liquids (but even aqueous media) in place of anhydrous VOCs, has just made a huge breakthrough among chemical practitioners. ${ }^{[6]}$ The question is especially relevant in the case of highly reactive organometallic compounds such as Grignard and organolithium reagents. Infact, protic media and hydrous conditions are often considered the natural foes of these organometallic reagents, and the lion's share of their reactions is (still) traditionally carried out in dry organic solvents, with strict moisture exclusion (that is under an inert atmosphere), and often employing low temperatures.

The object of this Concept article is to showcase recent progress of polar organometallic chemistry, and in particular of the reactivity of compounds of s-block elements, in aqueous surroundings, when using water as the only reaction medium, and in deep eutectic solvents. Structural, mechanistic and synthetic aspects of this chemistry will be discussed highlighting selected, key papers. We hope that this Concept article is helpful to those interested in carrying out organometallic reactions in unconventional, bio-inspired solvents, and encourages future research and deepening in this exciting area The interested reader is also encouraged to consult our previous review in this field. ${ }^{[6]}$

\section{The Impact of Water on the Reactivity of Polar Organometallic Compounds}

What is water? Water is indisputably a life force for all human beings as is vital to life on Earth. It is not only the solvent of choice for Nature to carry out biosyntheses, but also an attractive solvent in the development of green and environmentally safe processes because of its unique physical and chemical properties when compared to traditional organic solvents: it is nonflammable, non toxic, inexpensive, and acts as an excellent heat sink because of its large heat capacity. For these reasons, the field of "aqueous organic synthesis" is mushroomed with excellent papers and reviews written year in, year out; ${ }^{[7]}$ the colligative structure and catalytic properties of water are a burgeoning and hot field of investigation as well. ${ }^{[8]}$ 
One of the most intriguiguing properties of water is its peculiar dynamic three-dimensional hydrogen-bonded network, which retains several of the structural characteristics of ice, but at the same time is characterised by high hydrogen-bond exchange rates. It is indeed thought that each molecule forms, on average, 3.5 hydrogen bonds with a lifetime on the order of 1-20 ps ${ }^{[9]}$ However, as pointed out by Steiner in an enlightening review, ${ }^{[10]}$ the hydrogen bond is a much broader phenomenon: "we know of hydrogen bonds that are so strong that they resemble covalent bonds in most of their properties, and we know of others that are so weak that they can hardly be distinguished from van der Waals interactions." As a matter of fact, 16 out of a possible 36 different types of $\mathrm{H}$-bonds have recently been identified and ordered in water according to their intrinsic strength, the number of strong $\mathrm{H}$-bonds increasing by increasing the temperature. ${ }^{[11]}$

As for the use of water as a medium in organic synthesis, several expressions have been introduced according to the solubility of the reacting species: "in water", for clear solutions of soluble organic reactants in water; "on water", for organic reactions between water insoluble reactants and taking place at the interface of a bulk water layer without any additive; and even "in the presence of water", for reaction proceeding in a biphasic system in the presence of a large amount of water. ${ }^{[7 e, f, 12]} A$ fluorishing field with an increasing number of publications is also represented by technologies that take advantage of micellar catalysis in the presence of engineered surfactants (e.g., TPGS750-M, Triton-X, etc.). ${ }^{[13]}$ The above considerations are important to gain a better understanding of the factors influencing the outcome of organometallic reactions when water is used as the reaction medium (vide infra). Indeed, an interplay of different effects may be operating depending on the greater or the lesser solubility of the reactants in water. ${ }^{77 e, f, 12]}$

It is well-known that water is of paramount importance in biological processes, and interactions with surrounding water is crucial for both three-dimensional folding and function of biomolecules. ${ }^{[14 a]}$ Can we parallel the role played by water in biomolecules with that played in organometallic compounds? Similarly, for the latter, intermolecular hydrogen bonding interactions with water result in self-organization, bond activation and thus in new carbon-carbon bond formation. ${ }^{[14 b]}$ However, when it comes to highly reactive organometallic compounds of sblock elements (mainly Grignard and organolithium reagents), whose utilisation typically requires strictly anhydrous and aprotic volatile organic solvents and inert atmosphere, ${ }^{[15]}$ the role played by water becomes less clear and questionable. A perusal of the literature reveals that the deliberate or adventitious addition of stoichiometric or catalytic amounts of water in organometallic chemistry sometimes proved to be crucial in redirecting reaction in interesting and unexpected ways: for example, by favouring a lithium/halogen exchange, by speeding up the reaction rate, or by dramatically increasing the enantiomeric excess in asymmetric reactions. ${ }^{[6]}$ The "water effect" with organometallic compounds, however, is not yet well understood, and evidence suggests that proton transfer from water even towards very strong basic centers is not a straightforward process as is commonly believed. In the presence of metal-organic compounds, even in dry solvents, proton transfer seems to be governed by complex dynamic equilibria and by the aggregation states of clusters involved (vide infra). Let's have a look, for example, at a surprising effect occurring in Li-enolate chemistry.

As shown by Seebach and co-workers, $\mathrm{Li}$ enolates generated from $\mathrm{LiNR}_{2}$ bases apparently form a "stable" complex with the sec-amine co-product $(\mathbf{1}$, Scheme 1$)$. Only under the influence of an electrophile (e.g., $\mathrm{D}^{+}$), the $\mathrm{NH}$-proton is "kicked back" to where it came from, thereby generating the starting, non-deuterated carbonyl compound (2, Scheme 1). The presence of such a kinetically inert complex was supported by the observation that the removal of the above proton by $n$-BuLi finally resulted in the successful trapping of the intermediate enolate with the electrophile, and in the formation of the desired product in good yield (3, Scheme 1). ${ }^{[16]}$ Water itself seems somewhat reluctant to act as a Lewis base as well even in the presence of strong electrophilic centers. For example, living carbocations generated in water by the formation of hydrogen bonds between water and the hydroxy groups of alcohols decorated with electron-donating substituents exhibit a lifetime of 10-20 s, and are thus suitable for direct nucleophilic substitutions by aromatic heterocycles without being first "quenched" by water! ${ }^{[17]}$

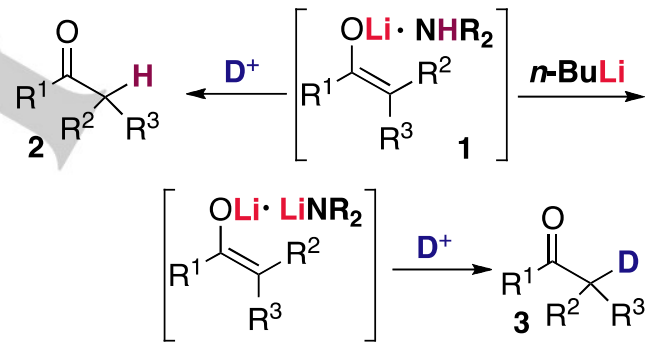

Scheme 1. Effects in Li-enolate chemistry.

An unexpected cooperation between $n$-BuLi and water was recently discovered by Merino and co-workers during the optimisation of the reaction conditions for the stereoselective synthesis of 2,5-disubstituted-3-oxazolines by reacting nitrones with aldehydes. These cycloadditions were found to take place smoothly, providing the expected adducts in very good yields, only in the presence of a catalytic amount (20 mol\%) of $n$-BuLi. Detailed mechanistic studies provided evidence for lithium-ion catalysis and supported an autocatalytic role played by water. The in-situ formed water, in turn, triggers a new catalytic round, and contributes to speed up the reaction even at low temperature. The use of molecular sieves, indeed, dramatically reduced the reaction rate. ${ }^{[18]}$

What is the aptitude of water to behave as a ligand of $\mathrm{Li}^{+}$in organolithium compounds? and in such an eventuality, how the reactivity of water is, in turn, modified? Gimbert and co-workers recently investigated this phenomenon by means of mass spectrometry and DFT computations by reacting the lithium amide of a 3-aminopyrrolidine with water. The authors noticed that the aggregation with strong polar partners such as $\mathrm{LiX}(\mathrm{X}=$ 
$\mathrm{Cl}, \mathrm{Br}$, or $\mathrm{Me}$ ) is responsible for the introduction of a waterprooftype character in the corresponding complex 4 (Scheme 2), thereby leaving the basic nitrogen amide untouched. Small selfassembled water clusters (up to $4 \mathrm{H}_{2} \mathrm{O}$ ) were computed to become, on the whole, increasingly coordinating for the lithium ion pulling it away from the basic center. ${ }^{[19]}$ The presence of LiX in the corresponding heteroaggregate also contributes to repel water from the nitrogen, thereby rendering its protonation more difficult. Interestingly, the transition state associated with the $\mathrm{N}$ protonation reaction does not correspond to the $\mathrm{N}$-protonation itself, but rather to the rotation of the molecule of water around the $\mathrm{Li}$ atom! These pieces of evidence support even more the idea that protonation of a basic center is a complex phenomenon influenced by both aggregation and the geometry of the organometallic species, but is also mediated by the oxophilicity of the cations involved.

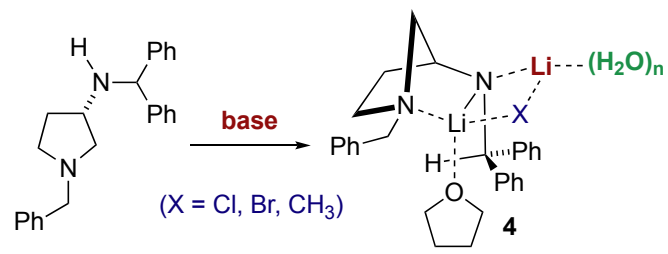

Scheme 2. Schematic structure of the lithium amide mixed aggregate 4

Stalke and co-workers recently succeeded in the crystallisation of a water-containing organopotassium complex, which surprisingly revealed to be strongly recalcitrant to hydrolysis. ${ }^{[20]}$ By treating a bis(benzoxazol-2-yl)methane $\mathbf{5}$ with $\mathrm{KH}$ in THF, in the presence of an incompletely dried 18-crown-6 ether, 6 crystallised with 0.35 equivalents of water and two THF molecules (Scheme 3).

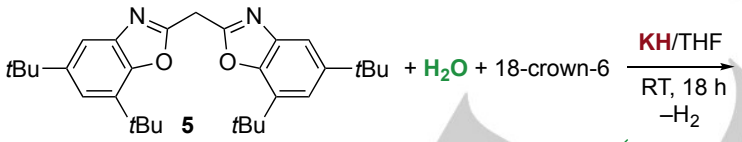

Scheme 3. Synthesis of water-containing organometallic potassium complex 6

Complex 6 is a rare example of a bis(benzoxazol-2-yl)methanide ligand displaying solely oxygen coordination to a metal ion despite the main electron density located at the nitrogen atom of the aromatic ring. The presence in the solid state of a crown ether molecule, and the peculiar geometry adopted by the ligand, allows the formation of a unique two-dimensional hydrogen- bonded water network at the surfaces of both the ligand and the ether. Such a chelated supramolecular network most probably is the key feature for an enhanced stability exhibited by 6 against hydrolysis. Indeed, careful NMR titration DOSY experiments revealed full protonation of complex $\mathbf{6}$ (after seven days and the addition of 114 equivalents of water) only in the presence of the crown ether. In its absence, it took $24 \mathrm{~h}$ and the addition of 7.60 equivalents of water in order for the complex to be fully protonated (Figure 1).

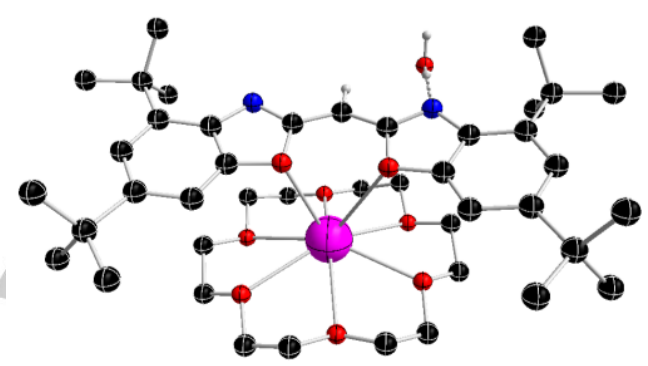

Figure 1. Molecular structure of complex 6 . Figure with atom labels attached

Can we replace dry VOCs with bulk water for carrying out reactions of highly polar organometallic compounds? Recently Capriati and co-workers first showed that when assorted aliphatic and aromatic Grignard reagents in THF solutions, or organolithium reagents in ethereal/hydrocarbon solutions (range concentration 0.5-3.0 M), were rapidly spread out over suspensions of sparingly soluble (ca. $10^{-3} \mathrm{~mol} \mathrm{~L}^{-1}$ ) aliphatic and aromatic enolizable $\gamma$-chloroketones 7 in water (tipically 0.5 $\mathrm{mmol}$ per $1 \mathrm{~mL}$ of water), under air at room temperature (RT) and under vigorous stirring, the expected tetrahydrofuran (THF) derivatives 8 straightforwardly formed in satisfactory yields (3 equiv.: up to $75 \%$; 6 equiv.: up to $85 \%$ ) and competitively with protonolysis upon final treatment with $10 \%$ aq. $\mathrm{NaOH}$ (Scheme 4). ${ }^{[21]}$

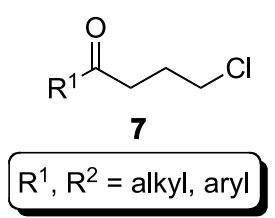

1) $R^{2} M, H_{2} \mathrm{O}$ $\underset{\text { 2) } 10 \% \text { aq. } \mathrm{NaOH}}{\stackrel{\text { under air, } \mathbf{R T}, 10 \mathrm{~min}}{\longrightarrow}}$

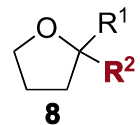

up to $85 \%$ yield

Scheme 4. Addition reaction of organometallic reagents to $\gamma$-chloroketones 7 "on water", under air and at room temperature, to afford THF derivatives 8.

Despite extensive theoretical studies, the "on water" phenomenon is not well understood yet. An important result reported by Sharpless and co-workers in their seminal paper, ${ }^{112 a]}$ and often overlooked in the literature, is a solvent isotope effect observed when $\mathrm{D}_{2} \mathrm{O}$ was used in place of water. Upon running nucleophilic additions depicted in Scheme 4 on- $\mathrm{D}_{2} \mathrm{O}$, only small deuterium isotope effects were observed. According to the 
model proposed by Jung and Marcus, trans-phase hydrogen bonding from dangling $\mathrm{OH}_{\text {free }}$ groups at the water interface to $\mathrm{H}$ bond acceptor sites on the organic reactants would be the main source of on-water catalysis as it contributes to stabilize organic transition states that are embedded in the water surface. ${ }^{[22]}$ As recently demonstrated by Ben-Amotz and co-workers, this is also a cooperative effect and the extent of $\mathrm{OH}_{\text {free }}$ groups at the interface water/hydrophobic surfaces depends either on the surface area involved or on the electrostatic nature of the surface itself. ${ }^{[23]}$ However, while, on the one hand, Marcus theory is consistent with the acceleration involving an interface with water, on the other hand, it does not account for the deuterium isotope effect. Thus, only mild on-water catalytic effects, as in the case above discussed, may be suggestive of hydrogen bonding. ${ }^{[24]}$ At the same time, the packing density of supramolecular clusters of water created by strong intermolecular hydrogen bonds may play a key role as well in making protons less available for protonolysis processes. The above nucleophilic additions, indeed, failed upon replacing water with an ambiphilic, protic solvent such as $\mathrm{MeOH}$ whose ability to undergo self-association through hydrogen bond is much more limited compared to water. ${ }^{[25]}$ Water does have a unique $\mathrm{H}$ bonding property and a unique surface structure that cannot be mimicked by any alcohol molecule. ${ }^{[23,26]}$

Throughout the years, several reactions have been reported to display strong on-water catalytic effects, and thus to exhibit significative primary deuterium isotope effects with a noticeably slower rate in $\mathrm{D}_{2} \mathrm{O}$. Examples include aza-Claisen rearrangements on organic nitrogen systems, ${ }^{[27 a]}$ displacements of $\mathrm{OH}$ groups at allylic and benzylic sites, ${ }^{[17,27 b]}$ epoxide ring opening in the absence of any added catalyst/acid, ${ }^{[27 c]}$ and the nucleophilic addition of formaldehyde hydrazones to readily available $\alpha$-keto esters. ${ }^{[27 d]}$ In order to explain such a significant solvent isotope effect, the Bettie and McErlean research groups have proposed that proton transfer (that is, acid-base chemistry) across the water-organic interface, rather then $\mathrm{H}$-bonding effects, may be involved in the rate-limiting step. ${ }^{[28]}$ Capriati and coworkers recently reported that the addition of either organolithium reagents to non-activated imines $\mathbf{9}$ or of highly polar organometallic compounds to nitriles $\mathbf{1 0}$ proceeds quickly and chemoselectively with a broad substrate scope, at RT, under air, and vigorous stirring, with water as the only reaction medium, thereby providing the expected secondary amines 11 and tertiary carbinamines 12 , respectively, in yields of up to and over $99 \%$ (Scheme 5). ${ }^{[29]}$

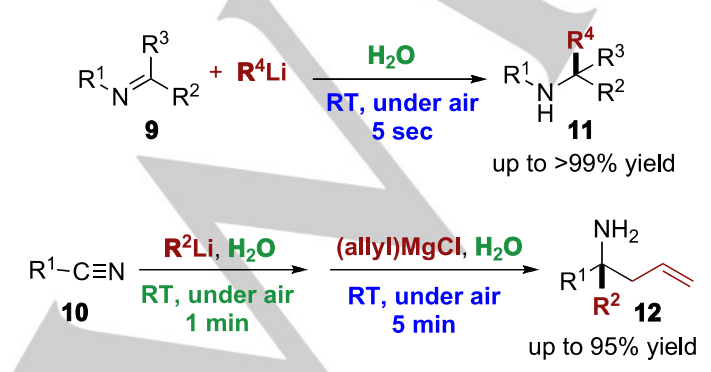

Scheme 5. Addition of organometallics to imines $\mathbf{9}$ and nitriles 10 "on water" affording amine derivatives 11 and tertiary carbinamines 12 , respectively.
Of note, (a) a significant solvent $\mathrm{D} / \mathrm{H}$ isotope effect was observed for the nucleophilic addition of $n$-BuLi to benzylidene anyline, as the yield in the corresponding adduct dropped down from $96 \%$ to $57 \%$ for the on $-D_{2} \mathrm{O}$ reaction; (b) the water quenching was not instantaneous as the expected adduct could still be recovered (with a $20 \%$ yield after $5 \mathrm{sec}$ in the model reaction) upon spreading $n$-BuLi over water followed by the addition of imine, (c) the addition to imines could be successfully scaled-up to a gram scale. Intriguingly, as for the two-step onepot synthesis of carbinamines, the addition of organolithiums and allyl Grignard reagents proved to be effective either in the case of poorly water-soluble (e.g., benzonitrile) or for watersoluble (e.g., acetonitrile) nitriles. Thus, the outcome of these reactions seems to be not strictly dependent on the water solubility of the substrate. This reinforces the hypothesis that the unique tridimensional molecular structure of water is the key for a proper understanding of the missing protonolysis processes. Other effects (e.g., the hydrophobic effect, hydrogen bonding effects, and water polarity effects), on the other hand, are known to govern "in-water" reactions. ${ }^{[7 \mathrm{~g}]}$

\section{Introducing Deep Eutectic Solvents to Polar Organometallic Chemistry}

Pionereed by Abbot in 2003, ${ }^{[30]}$ Deep Eutectic Solvents (DESs) are combinations of 2 or 3 safe, inexpensive, and natureinspired components able to engage in reciprocal hydrogen bond interactions to form a eutectic mixture with a melting point much lower than that of either individual component. ${ }^{[31]}$ Compared to conventional VOCs, DES show high thermal stability, non-flammability and practically no vapour pressure, therefore low volatility. ${ }^{[32]}$ Typical DES components (e.g., choline chloride, urea, natural carboxylic acids, amino acids and carbohydrates, polyalcohols) come from renewable sources. Thus, their biodegradability is extraordinarily high, and their toxicity is non-existent or very low. ${ }^{[33]}$ Furthermore, the atom economy on the DES formation is the highest possible as all initial components are included in the final mixture. All these factors have made DESs the chosen solvents in many areas, such as metal electrodeposition and electrochemistry, ${ }^{[34]}$ polymerisation and material sciences, ${ }^{[35]}$ extraction and separation processes, ${ }^{[36]}$ bio- and organocatalysis, ${ }^{[37]}$ solar technology and photosynthesis, ${ }^{[38]}$ whereas their applications in metal-mediated synthetic transformations using polar organometallics have remained virtually unexplored as the canon of this chemistry is incompatibility with protic solvents. ${ }^{[6]}$

Spotting a gap in potential, Hevia and Garcia-Alvarez have reported the chemoselective alkylation of ketones 13 by RMgX and $\mathrm{RLi}$ reagents, at room temperature, under air, in watercontaining $D E S$, a trio of conditions seemingly incompatible with polar organometallics. Yet, incredibly, these conditions offer improved yields and better selectivities than standard inert atmosphere protocols. These reactions work well for a range of organometallic reagents and for both aliphatic and aromatic ketones, thereby providing tertiary alcohols 14 in up to $90 \%$ yield (Scheme 6). ${ }^{[39]}$ 


$$
\begin{aligned}
& \mathrm{R}^{1}, \mathrm{R}^{2}=\text { aryl or alkyl; } \mathrm{R}^{3}=\text { vinyl, alkyl, ethynyl, } \mathrm{Ph}, \mathrm{Bu} \\
& \mathrm{M}=\mathrm{Li}, \mathrm{MgBr}
\end{aligned}
$$

Scheme 6. Addition reactions of organometallic reagents to ketones 13 in DES $\left(1 \mathrm{ChCl} / 2 \mathrm{H}_{2} \mathrm{O}\right)$, at room temperature under air, to give tertiary alcohols 14 .

By comparing the reactivity profiles of these polar organometallics (RLi or RMgX) in DESs and in water, it was suggested that the formation of kinetically activated mixedammonium "ate" salts takes place in choline chloride $(\mathrm{ChCl})$ based DESs by co-complexation of the above reagents with the ammonium salt $\mathrm{ChCl}$, which is the $\mathrm{H}$-bond acceptor component of the DES studied. This hypothesis is consistent with the isolation and the structural characterisation of complex 15 $\left[\left\{\mathrm{NBu}_{4}\right\}^{+}\left\{\mathrm{Mg}\left(\mathrm{CH}_{2} \mathrm{SiMe}_{3}\right) \mathrm{Cl}_{2}(\mathrm{THF})\right\}^{-}\right]$as a result of the reaction of Grignard reagent $\mathrm{Me}_{3} \mathrm{SiCH}_{2} \mathrm{MgCl}$ with tetrabutylammonium chloride in THF (Figure 2). ${ }^{[39]}$
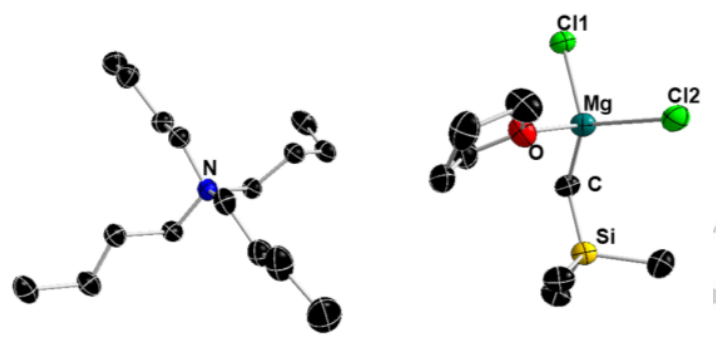

Figure 2. Molecular structure of complex 15.

Thus, it appears that $\mathrm{ChCl}$ has a double role in these reactions, being not only a constituent of the $D E S$, but also a source of halide for the organometallic reagent. The profound activating effect that inorganic salts such as $\mathrm{LiCl}$ have when added to s-block metal reagents is best exemplified by Collum's studies on the reactivity of LDA. In this case, by adding as little as $1 \mathrm{~mol} \%$ of $\mathrm{LiCl}$, its 1,4 -addition to unsaturated esters occurs 70 times faster ${ }^{[40]}$ Along with this suggested anionic activation of the organometallic reagent, generating a powerful nucleophilic species that reacts preferentially with the unsaturated organic substrate instead of undergoing protonolysis, other key aspects need to be taken into consideration. These include the influence of the strong $\mathrm{H}$-bond network present in DESs, which can also contribute either in shielding the organometallic reagent from the competitive protonolysis or in activating the carbonyl compound by $\mathrm{H}$-bonding interactions. ${ }^{[6]}$

Despite the possibility of fine-tuning physico-chemical properties of $D E S s$ by varying the nature and the molar ratio of their components, ${ }^{[33]}$ the liquid structures of these eutectics still remain largely unknown. Filling this gap in the knowledge, Edler has shrewdly applied neutron diffraction and atomistic modelling to establish the liquid structure of reline, one of the most widely used DES which is made up by a $1: 2$ mixture of $\mathrm{ChCl}$ and urea. ${ }^{[41]}$ These studies have revealed the formation of a strong hydrogen-bonding network within the liquid, with significant ordering interactions not only between urea and the chloride anion but also between choline (via its $\mathrm{OH}$ group) and the chloride anion. In other words, a complex sandwich structure forms where choline and urea bond synergistically to the chloride anion while maximising their own weaker interactions. More recently, Edler has also investigated by neutral total scattering the nanostructure of reline across a wide hydration range reporting that DES nanostructure is retained up to $42 \mathrm{wt} \%$ $\mathrm{H}_{2} \mathrm{O}$. At a higher water percentage, solvophobic sequestration of water into the nanostructure domain of reline becomes unfavourable, and the DES-water mixture is better described as an aqueous solution of $D E S$ components. ${ }^{[42]}$

While understanding solvent effects in organolithium chemistry has played an instrumental role in the development of novel applications of these commodity reagents in synthesis, ${ }^{[15]}$ the structures of polar organometallics in DESs and their interactions with the different components of these solvents still remain a black box. Thus, advancing the fundamental knowledge on DES structuring, and solvent-reagent interactions will be the key in order to make further progress in the synthetic applications of polar organometallics in these unconventional solvents. More recently, by expanding the scope of these type chemistry in DESs, Hevia and Garcia-Alvarez have reported the addition reaction of a wide range of organolithium reagents to non-activated imines. ${ }^{[43]}$ In this study, better conversions are observed using a 1:2 mixture of $\mathrm{ChCl}$ and glycerol (Gly) (Scheme 7). Addition reactions take place much faster than the competing protonolysis process, giving rise to the desired secondary amines 17: $i$ ) in good yields (73-95\%); ii) after short reaction times (3 s); iii) under air and at room temperature; and iv) with high selectivity (only unreacted imine and the expected amine were observed in the reaction crudes). Similarly to what observed using water as the reaction medium (Scheme 5), ${ }^{[29]}$ also in this case, factors like the strong tridimensional molecular structure of hydrogen bonds of DES (which parallels that of water) and an efficient activation (by intermolecular hydrogen bonds) towards nucleophilic addition of the azomethine moiety provided by $D E S$ itself could be key points to explain the observed high chemoselectivity.

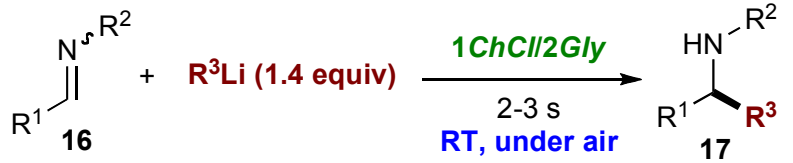

$$
\begin{aligned}
& \mathrm{R}^{1}, \mathrm{R}^{2}=\text { aryl or alkyl; } \mathbf{R}^{3}=\text { alkyl, aryl } 73-95 \% \text { yield }
\end{aligned}
$$

Scheme 7. Addition of organolithium reagents $(R L i)$ to imines 16 at room temperature, in the presence of air and in the eutectic mixture $\mathrm{ChCl/glycerol,}$ to give secondary amines 17

Moreover, this study has also assessed an unusual kinetic stability of $n$-BuLi in the unique tridimensional molecular structure of $D E S$ by reversing the order of addition of the reagents (Scheme 8 ). Indeed, upon first adding $n$-BuLi to the 
ChC/Gly eutectic mixture prior to the addition of the $\mathrm{N}$ benzylideneaniline, the observed yield of the final expected amine is above $50 \%$ even after 1 min of interval, at room temperature and in the presence of air. It takes at least $3.5 \mathrm{~min}$ for the reactivity of $n$-BuLi towards $N$-benzylideneaniline to be totally supressed.
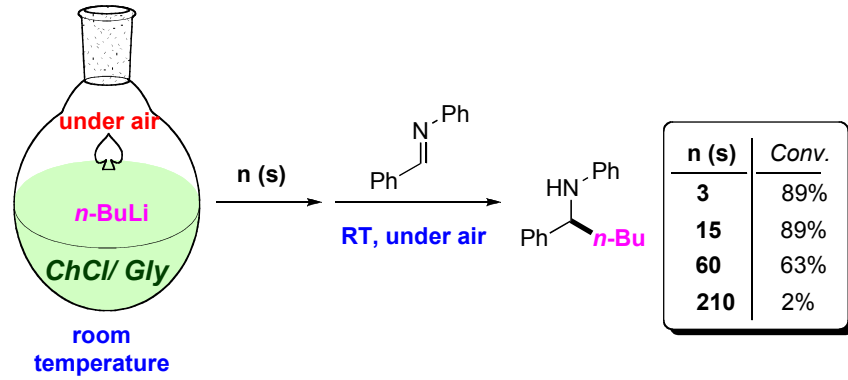

Scheme 8. Assessing the stability of $n$-BuLi in the eutectic mixture ChC//Gly.

Contemporaneously and independently from these studies, Capriati and co-workers have corroborated the feasibility of the employment of organolithium reagents in DESs for metalmediated organic transformations. A regioselective desymmetrisation of diaryltetrahydrofuran 18 was successfully achieved by exploiting a THF-directed ortho-lithiation reaction promoted by $t$-BuLi in cyclopentyl methyl ether (CPME) or $\mathrm{Et}_{2} \mathrm{O}$ to generate the ortho-lithiated intermediate 19. The latter was then intercepted with several electrophiles (both addition reactions and nucleophilic substitutions) in $\mathrm{ChCl}$-based eutectic

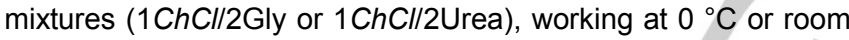
temperature and under open air conditions, providing the expected adducts $\mathbf{2 0}$ in yields of up to $90 \%$, and competitively with protonolysis (Scheme 9). ${ }^{[4]}$

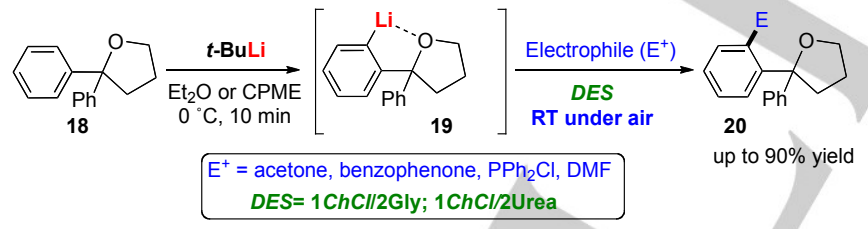

Scheme 9. Ortho-lithiation of diaryltetrahydrofuran 18 and concomitant functionalisation with different electrophiles in protic eutectic mixtures.

Furthermore, investigations on the metalation of these systems has led to the discovery that, as the result of a directed lateral lithiation, o-tolyltetrahydrofuran derivatives 21 can undergo a highly regioselective ring-opening transformation with concomitant formation of new $\mathrm{C}-\mathrm{C}$ bonds which grants access to functionalised primary alcohols $\mathbf{2 2}$ (Scheme 10). This reaction is triggered by organolithium reagents such as $s$-BuLi, $i$-PrLi and $t$ BuLi (overall 2 equiv) and can be successfully executed in ethereal, Gly-based eutectic mixtures as benign reaction media, at $0{ }^{\circ} \mathrm{C}$ and under air, thereby providing alcohols 22 in up to and over $98 \%$. These feature the incorporation in their skeletons both of a second equivalent of base and of an electrophile at a tertiary carbon atom. ${ }^{[45]}$ These findings highlight the potential of
$D E S s$ to carry out multistep synthetic transformations where the kinetic reactivity of the organolithium reagents favour this novel metalation/C-C bond formation/C-O bond breaking process over their degradation in the presence of air, moisture and glycerol.
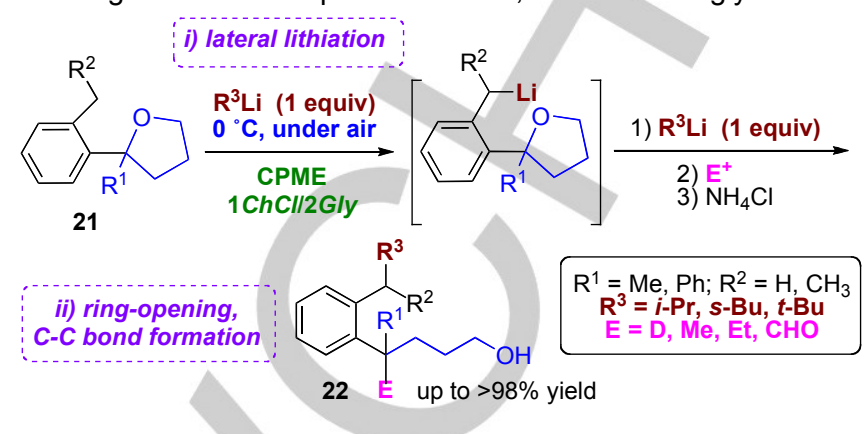

Scheme 10. Multistep benzylic lithiation/ring-opening of o-tolyltetrahydrofurans 21, promoted by RLi reagents in ethereal/eutectic mixture reaction media and in the presence of air, to give functionalised alcohols 22 .

During the last years, a tremendous advance has been made in the field of transition-metal-catalysed organic reactions in DESs as environmentally friendly reaction media, with broad application in well-established synthetic procedures ranging from $\mathrm{Pd}$-catalysed cross-coupling processes to Cu-catalysed Click Chemistry reactions (CuAAC), among others. ${ }^{[46]}$ Building on previous findings, ${ }^{[47]}$ Capriati, García-Álvarez and co-workers have successfully assembled the Ru-catalysed redox isomerisation of allylic alcohols $\mathbf{2 3}$ with the chemoselective addition of organolithium reagents to the in-situ formed ketones 24 en-route to tertiary alcohols 25 in DESs (Scheme 11). ${ }^{[48]}$

The overall one-pot sequential transformation, run in $\mathrm{ChCl}$ based eutectic mixtures, formally involves the following three steps: $i)$ the reduction of a $\mathrm{C}-\mathrm{C}$ double bond; ii) the oxidation of a secondary carbinol moiety; and iii) the chemoselective addition of the RLi reagent at room temperature and in the presence of air. The desired alcohols $\mathbf{2 5}$ have been straightforwardly synthesized in up to $99 \%$ yield without the need of isolation/purification of any intermediate, and in the absence of tedious/intricate compartmentalisation procedures. Remarkably, this tandem protocol can also successfully be performed using also pure water as the reaction medium. ${ }^{[48]}$

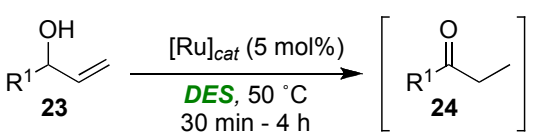

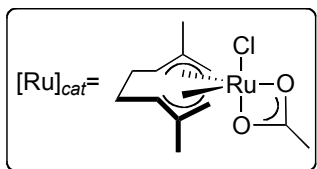

$\mathrm{R}^{1}=$ alkyl, ary

$\mathrm{R}^{2}=n-\mathrm{Bu}, \mathrm{s}-\mathrm{Bu}, \mathrm{Me}, \mathrm{Ph}$
$\mathrm{R}^{2} \mathrm{Li} \mid \begin{gathered}\text { DES, } 3 \mathrm{~s} \\ \mathrm{RT} \text {, under air }\end{gathered}$

$\mathrm{OH}$<smiles>[R]C([R])(O)CC</smiles>

25

up to $>99 \%$ yield
Scheme 11. Synthesis of tertiary alcohols 25 via a one-pot combination of Rucatalysed redox isomerisation of allylic alcohols 23 with concomitant addition of $\mathrm{RLi}$ reagents in $\mathrm{ChCl}$-based eutectic mixtures. 
While most of these DES studies use solvent systems based on the non-toxic quaternary ammonium salt $\mathrm{ChCl}$, recent studies on the addition of aryllitium reagents to nitriles 26 to access aromatic ketones $\mathbf{2 7}$, under air and at room temperature, have shown that glycerol can also be used as an environmentally responsible reaction medium, showing similar conversions to those observed using the eutectic mixture ChCl/2Gly or water (Scheme 12). ${ }^{[49]}$ Interestingly, these reactions occur heterogeneously ("on glycerol" conditions) where the lack of solubility of nitriles in glycerol and the ability of this solvent to form strong intermolecular $\mathrm{H}$-bonds seem to be key factors to allow the smooth nucleophilic addition of the ArLi reagents to these carboxylic acid derivatives. Indeed, when glycerol-soluble $\mathrm{MeCN}$ was employed, no reaction was observed. Consistent with the proposed "on glycerol" conditions, with the reactions being thought to occur at the organic/liquid glycerol interface with glycerol-insoluble reactants, the yields are greatly influenced by stirring/agitation effects, which can affect the volume and surface area of the organic droplets (Scheme 12). This reactivity contrasts with that observed using water as the reaction medium, where even water-soluble nitriles could be successfully elaborated to tertiary carbinamines by a double nucleophilic addition jointly promoted by $\mathrm{ArLi}$ and (allyl) $\mathrm{MgCl}$ (Scheme 5). ${ }^{[29]}$ Thus, although sharing some physico-chemical properties with water, glycerol is on a different ground, and a comparison of the outcome of the reaction using these two solvent systems is always recommended.

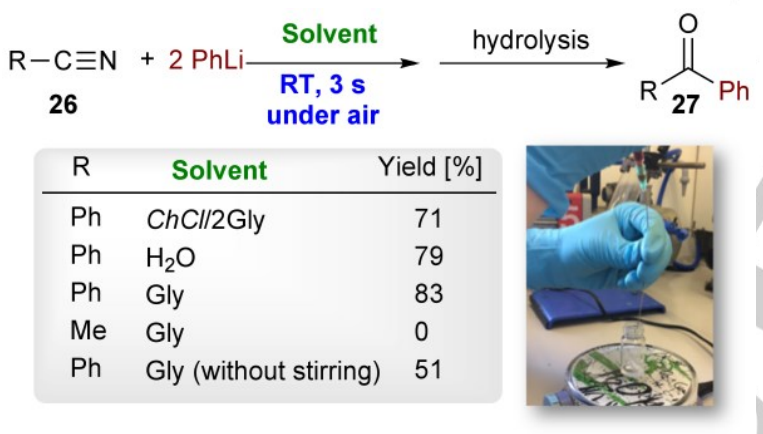

Scheme 12. Addition of PhLi to nitriles 26 at room temperature, in the presence of air and using non-conventional solvents, to give aromatic ketones 27.

\section{Conclusions and Outlook}

As today's world requests greener, more sustainable, and safer chemical transformations to perfect the use of polar organometallics, replacing toxic volatile organic solvents by more environmentally benign, bio-inspired solvents (e.g., water, glycerol, deep eutectic solvents), working at the same time under air and at room temperature, constitutes one of the most momentous challenges in organometallic chemistry. While for decades these reaction conditions have been considered disallowed, exciting new studies discussed in this Concept article suggest that this is not an insurmountable challenge.
Hints that the impossible may become possible have started to emerge, unlocking a myriad of opportunities for further development of sustainable, air and moisture compatible maingroup-metal-mediated organic synthesis. This is extremely important as it establishes a step change in the way that organometallic synthesis can be carried out from now onward. An interplay of factors represented by complex dynamic equilibria and the aggregation states of the organometallic species involved, strong $\mathrm{H}$-bonded networks, the formation of competitive, more nucleophilic "ate" complexes, and catalytic effects at the organic/solvent interface seems to be responsible for the outcome and the acceleration these s-block-metalmediated reactions undergo in the above unconventional reaction media. Thus, each reaction needs to be discussed on a case-by-case basis. Profound advances in the knowledge and in the understanding on a molecular level of polar organometallic reactions performed in strongly hydrogen-bond associated mixtures will also provide the rationale for planning tailored transformations in environmentally responsible reaction media never thought possible before. The idea of building an easily tunable, structurally confined chiral bio-based reaction media for running stereoselective metal-catalysed and metal-mediated reactions is certainly the ultimate goal worthy pursuing in this field. Exploring the reactivity of new, polar organobimetallic complexes both in water and in safe, green and bio-renewal $D E S s$, under stoichiometric conditions but also in catalytic regimes, may also open the floodgates to unprecedented synthetic methodologies in terms of versatility and chemoselectivity, thereby creating new opportunities in fine chemicals production and catalysis, but at the same time minimising chemical hazards.

\section{Acknowledgements}

This work was financially supported by the University of Bari (codes: Perna01333214Ricat; Capriati005057Prin15), and by the Interuniversities Consortium C.I.N.M.P.I.S. (project code: COCM8470P0) and by the EPSRC (grant number EP/N011384/1) Professor Dieter Seebach (ETH University) is also gratefully acknowledged for enlightening discussion.

Keywords: sustainable chemistry • polar organometallics $\bullet$ deep eutectic solvents $\bullet$ water chemistry $\bullet$ solvent effects

[1] P. T. Anastas, J. C. Warner, Green Chemistry: Theory and Practice, Oxford University Press, New York, 1998

[2] a) Green Techniques for Organic Synthesis and Medicinal Chemistry (Eds.: W. Zhang, B. W. Cue, Jr.), Wiley-VCH, Weinheim, Germany, 2012; b) K. Shanab, C. Neudorfer, E, Shirmer, H. Spreitzer, Curr. Org. Chem. 2013, 17, 1179-1187; c) C. P. Ashcroft, P. J. Dunn, J. D. Hayler, A. S. Wells, Org. Process Res. Dev. 2015, 19, 740-747; d) P. J. Dunn, A. S. Wells, M. T. Williams, Future Trends for Green Chemistry in the Pharmaceutical Industry in Green Chemistry in the Pharmaceutical Industry (Eds.: P. J. Dunn, A. S. Wells, M. T. Williams), Wiley-VCH, Weinheim, Germany, 2010, pp. 333-355.

[3] a) K. R. Seddon, Kinetics and Catalysis 1996, 37, 693-697; b) B. H Lipshutz, S. Ghorai, Green Chem. 2014, 16, 3660-3679; c) B. H. 
Lipshutz, F. Gallou, S. Handa, ACS Sustainable Chem. Eng. 2016, 4 5838-5849; d) Bio-Based Solvents (Eds.: F. Jérôme, R. Luque), John Wiley \& Sons Ltd, 2017.

[4] C. Reichardt, Solvents and Solvent Effects in Organic Chemistry, Wiley-VCH, Weinheim, Germany, 2003.

[5] http://sci2017.org (accessed on 07.02.2018).

[6] J. García-Álvarez, E. Hevia, V. Capriati, Eur. J. Org. Chem. 2015, 6779-6799.

[7] a) U. M. Lindström, Chem. Rev. 2002, 102, 2751-2771; b) C.-J. Li, Chem. Rev. 2005, 105, 3095-3166; c) U. M. Lindström in Organic Reactions in Water: Principles, Strategies and Applications, 1st ed. Blackwell Publishing, Oxford, UK, 2007; d) K. H. Shaughnessy, Chem. Rev. 2009, 109, 643-710; e) C.-J. Li in Handbook of Green Chemistry, Reactions in Water, vol. 5 (Eds.: P. T. Anastas, C.-J. Li), Wiley-VCH Weinheim, Germany, 2010; f) A. Chanda, V. V. Fokin, Chem. Rev. 2009, 109, 725-748; g) R. N. Butler, A. G. Coyne, Chem. Rev. 2010 $110,6302-6337$.

[8] a) J. D. Smith, C. D. Cappa, K. R. Wilson, B. M. Messer, R. C. Cohen, R. J. Saykally, Science 2004, 306, 851-853; b) E. Vöhringer-Martinez, B. Hansmann, H. Hernandez-Soto, J. S. Francisco, J. Troe, B. Abel, Science 2007, 315, 497-501; c) W. J. Smit, H. J. Bakker, Angew. Chem. Int. Ed. 2017, 56, 15540-15544; Angew. Chem. 2017, 129 15746-15750

[9] F. Franks in Water - A Matrix of Life (2nd Ed.), Royal Society of Chemistry, Cambridge/U.K., 2000; b) I. Ohmine, S. Saito, Acc. Chem Res. 1999, 32, 741-749; c) R. Kumar, J. R. Schmidt, J. L. Skinner, Chem. Phys. 2007, 126, 204107.

[10] T. Steiner, Angew. Chem. Int. Ed. 2002, 41, 48-76; Angew. Chem. 2002, 114, 50-80.

[11] Y. Tao, W. Zou, J. Jia, W. Li, D. Cremer, J. Comput. Chem. 2018, 39, 293-306.

[12] a) S. Narayan, J. Muldoon, M. G. Finn, V. V. Fokin, H. C. Kolb, K. B. Sharpless, Angew. Chem. Int. Ed. 2005, 44, 3275-3279; Angew. Chem 2005, 117, 3339-3343; b) Y. Hayashi, Angew. Chem. Int. Ed. 2006, 45 8103-8104; Angew. Chem. 2006, 118, 8281-8282; c) Y.J. Zuo, J. Qu, J. Org. Chem. 2014, 79, 6832-6839.

[13] a) B. H. Lipshutz, S. Ghorai, G. T. Aguinaldo, Adv. Synth. Catal. 2008 350, 953-956; b) B. H. Lipshutz, A. R. Abela, Org. Lett. 2008, 10 5329-5332; c) C. M. Gabriel, N. R. Lee, F. Bigorne, P. Klumphu, M. Parmentier, F. Gallou, B. H. Lipshutz, Org. Lett. 2017, 19, 194-197; d) B. H. Lipshutz, F. Gallou, S. Handa, ACS Sustainable Chem. Eng. 2016 4, 5838-5849

[14] a) P. Ball, PNAS 2017, 114, 13327-13335; b) H. W. Roesky, M. G. Walawalkar, R. Murugavel, Acc. Chem. Res. 2001, 34, 201-211.

[15] a) H. J. Reich, Chem. Rev. 2013, 113, 7130-7178; b) V. Capriati, F. M. Perna, A. Salomone, Dalton Trans. 2014, 43, 14204-14210; c) E. Carl, D. Stalke, in Lithium Compounds in Organic Synthesis - From Fundamentals to Applications (Eds.: R. Luisi, V. Capriati), Wiley-VCH 2014, ch. 1, p. 3; d) U. Wietelmann, J. Klett, Z. Anor. Allg. Chem. 2018, $644,194-204$

[16] a) T. Laube, J. D. Dunitz, D. Seebach, Helv. Chim. Acta 1985, 68, 1373-1393; b) D. Seebach, Isr. J. Chem. 2017, 57, 55-65.

[17] P. G. Cozzi, L. Zoli, Angew. Chem. Int. Ed. 2008, 47, 4162-4166; Angew. Chem. 2008, 120, 4230-4234.

[18] V. Juste-Navarro, I. Delso, T. Tejero, P. Merino, Chem. Eur. J. 2016, 22, 11527-11532

[19] Y. Gimbert, D. Lesage, C. Fressigné, J. Maddaluno, J. Org. Chem. 2017, 82, 8141-8147.

[20] I. Koehne, S. Bachmann, R. Herbst-Irmer, D. Stalke, Angew. Chem. Int. Ed. 2017, 56, 15141-15145; Angew. Chem. 2017, 129, 15337-15342.

[21] L. Cicco, S. Sblendorio, R. Mansueto, F. M. Perna, A. Salomone, S. Florio, V. Capriati, Chem. Sci. 2016, 7, 1192-1199.

[22] Y. S. Yung, R. A. Marcus, J. Am. Chem. Soc. 2007, 129, 5492-5502.

[23] J. G. Davis, B. M. Rankin, K. P. Gierszal, D. Ben-Amotz, Nat. Chem. 2013, 5, 796-802.
[24] a) R. N. Butler, A. G. Coyne, J. Org. Chem. 2015, 80, 1809-1817; b) R N. Butler, A. G. Coyne, Org. Biom. Chem. 2016, 14, 9945-9960.

[25] a) S. Dixit, A. K. Soper, J. L. Finney, J. Crain, Europhys. Lett. 2002, 59 , 377-383; b) I. A. Beta, C. M. Sorensen, J. Phys. Chem. A 2005, 109 7850-7853.

[26] a) W. Gan, Z. Zhang, R. R. Feng, H. F. Wang, J. Phys. Chem. C 2007 111, 8726; b) M. Matsugami, R. Yamamoto, T. Kumai, M. Tanaka, T. Umecky, T. Takamuku, J. Mol. Liq. 2016, 217, 3-11.

[27] a) K. D. Beare, C. S. P. McErlean, Org. Biom. Chem. 2013, 11, 24522459; b) F.-Z. Zhang, Y. Tian, G.-X. Li, J. Qu, J. Org. Chem. 2015, 80 1107-1115; c) Z.-B. Xu, J. Qu, Chem. Eur. J. 2013, 19, 314-323; d) S Hajra, S. S. Roy, S. M. Aziz, D. Das, Org. Lett. 2017, 19, 4082-4085.

[28] J. K. Beattie, C. S. P. McErlean, C. B. W. Phippen, Chem. Eur. J. 2010 16, 8972-8974.

[29] G. Dilauro, M. Dell'Aera, P. Vitale, V. Capriati, F. M. Perna, Angew. Chem. Int. Ed. 2017, 56, 10200-10203; Angew. Chem. 2017, 129, 10334-10337.

[30] A. P. Abbott, G. Capper, D. L. Davies, R. K. Rasheed, V. Tambyrajah Chem. Commun. 2003, 70-71.

[31] Q. Zhang, K. De Oliveira Vigier, S. Royer, F. Jérôme, Chem. Soc. Rev. 2012, 41, 7108-7146.

[32] J. Naser, F. S. Mjalli, Z. S. Gano, J. Chem. Eng. Data. 2016, 61, 16081615

[33] M. Francisco, A. van den Bruinhorst, M. C. Kroon, Angew. Chem. Int Ed., 2013, 52, 3074-3085; Angew. Chem. 2013, 125, 3152-3163.

[34] a) E. L. Smith, A. P. Abbott, K. S. Ryder, Chem. Rev. 2014, 114 11060-11082; b) L. Milia, V. Dall'Asta, C. Ferrara, V. Berbenni, E. Quartarone, F. M. Perna, V. Capriati, P. Mustarelli, Solid State Ionics 2018, 323, 44-48

[35] a) D. Carriazo, M. C. Serrano, M. C. Gutiérrez, M. L. Ferrer, F. de Monte, Chem. Soc. Rev. 2012, 41, 4996-5014; b) F. del Monte, D Carriazo, M. C. Serrano, M. C. Gutiérrez, M. L. Ferrer, ChemSusChem 2014, 7, 999-1009.

[36] a) F. Pena-Pereira, J. Namieśnik, ChemSusChem 2014, 7, 1784-1800; b) B. Tang, H. Zhang, K. H. Row, J. Sep. Sci. 2015, 38, 1053-1064.

[37] a) R. A. Sheldon, Chem. Eur. J. 2016, 22, 12984-12999; b) P. Vitale, V M. Abbinante, F. M. Perna, A. Salomone, C. Cardllicchio, V. Capriati Adv. Synth. Catal. 2017, 359, 1049-1057; c) P. Vitale, F. M. Perna, G. Agrimi, I. Pisano, F. Mirizzi, R. V. Capobianco, V. Capriati, Catalysts 2018, 8, 55; d) L. Cicco, N. Ríos-Lombardía, M. J. Rodríguez-Álvarez, F. Moris, F. M. Perna, V. Capriati, J. García-Álvarez, J. González-Sabín, Green Chem. 2018, DOI:XXXX; e) C. R. Muller, I. Meiners, P. Domínguez de María, RSC Adv. 2014, 4, 46097-46101; f) E. Massolo, S. Palmieri, M. Benaglia, V. Capriati, F. M. Perna, Green Chem. 2016, 18, 792-797; g) R. Martínez, L. Berbegal, G. Guillena, D. J. Ramón, Green Chem. 2016, 18, 1724-1730; h) D. Brenna, E. Massolo, A Puglisi, S. Rossi, G. Celentano, M. Benaglia, V. Capriati, Beistein J. Org. Chem. 2016, 12, 2620-2626.

[38] a) C. L. Boldrini, N. Manfredi, F. M. Perna, V. Trifiletti, V. Capriati, A Abbotto, Energy Technol. 2017, 5, 345; b) F. Milano, L. Giotta, M. R Guacito, A. Agostiano, S. Sblendorio, L. Valli, F. M. Perna, L. Cicco, M. Trotta, V. Capriati, ACS Sustainable Chem. Eng. 2017, 5, 7768-7776.

[39] C. Vidal, J. García-Álvarez, A. Hernán-Gómez, A. R. Kennedy, E. Hevia, Angew. Chem. Int. Ed. 2014, 53, 5969-5973; Angew. Chem 2014, 126, 6079-6083.

[40] A. C. Hopeker, L. Grupta, Y. Ma, M. F. Faggin, D. B. Collum, J. Am. Chem. Soc. 2011, 133, 7135-7151.

[41] O. S. Hammond, D. T. Brown, K. J. Edler, Green Chem. 2016, 18 2736-2744.

[42] O. S. Hammond, D. T. Brown, K. J. Edler, Angew. Chem. Int. Ed. 2017 56, 9782-9785; Angew. Chem. 2017, 129, 9914-9917.

[43] C. Vidal, J. García-Álvarez, A. Hernán-Gómez, A. R. Kennedy, E. Hevia, Angew. Chem. Int. Ed. 2016, 55, 16145-16148; Angew. Chem. 2016, 128, 16379-16382. 
[44] V. Mallardo, R. Rizzi, F. C. Sassone, R. Mansueto, F. M. Perna, A. Salomone, V. Capriati, Chem. Commun. 2014, 50, 8655-8658.

[45] F. C. Sassone, F. M. Perna, A. Salomone, S. Florio, V. Capriati, Chem. Commun. 2015, 51, 9459-9462.

[46] For recent reviews in this field, see: a) J. García-Álvarez, Eur. J. Inorg Chem. 2015, 5147-5157; b) D. A. Alonso, A. Baeza, R. Chinchilla, G Guillena, I. M. Pastor, D. J. Ramón, Eur. J. Org. Chem. 2016, 612-632.
[47] C. Vidal, F. J. Suárez, J. García-Álvarez, Catal. Commun. 2014, 44, 76-79.

[48] L. Cicco, M. J. Rodríguez-Álvarez, F. M. Perna, J. García-Álvarez, V. Capriati, Green Chem. 2017, 19, 3069-3077.

[49] M. J. Rodríguez, J. García-Álvarez, M. Uzelac, M. Fairley, C. T. O’Hara, E. Hevia, Chem. Eur. J. 2018, 24, 1720-1725 


\section{Sustainable Polar Organometallic Chemistry}

\section{CONCEPT}

Make the switch! This Concept article highlights key developments of the chemistry of s-block elements in water and in bio-based solvents (glycerol and deep eutectic solvents), analyzing and discussing data in light of progresses made over the last few years.

Layout 2:

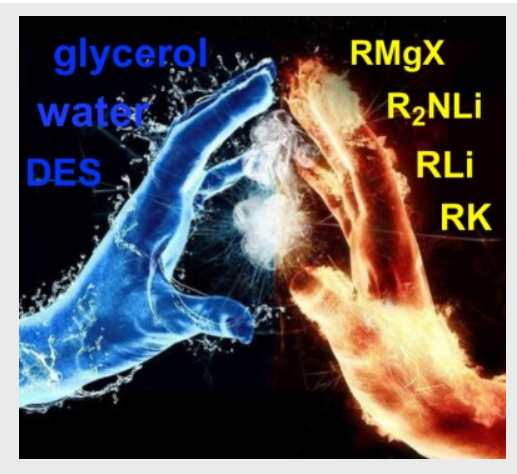

Joaquin García-Álvarez, * Eva Hevia, * and Vito Capriati*

Page No. - Page No.

The Future of Polar Organometallic Chemistry Written in Bio-Based Solvents and Water

\section{CONCEPT}

((Insert TOC Graphic here))
Author(s), Corresponding Author(s)*

Page No. - Page No.

Title 